\title{
Ciência \& Saúde Coletiva - 25 anos: contribuição para os estudos sobre gestação, parto e infância
}

\author{
Ciência \& Saúde Coletiva - 25 years: \\ contributions to pregnancy, delivery, and childhood studies
}

Janaína Calu Costa (https://orcid.org/0000-0002-7912-8685) ${ }^{1}$

Maria Fatima Santos Maia (https://orcid.org/0000-0001-6688-2745) ${ }^{1}$

Cesar Gomes Victora (https://orcid.org/0000-0002-2465-2180) ${ }^{1}$

${ }^{1}$ Centro Internacional de Equidade em Saúde, Programa de Pós-Graduação em Epidemiologia, Universidade Federal de Pelotas. R. Marechal Deodoro 1160, Centro. 96020-220 Pelotas RS Brasil.jcosta@equidade.org ${ }^{2}$ Universidade Federal do Rio Grande. Rio Grande RS Brasil.

\begin{abstract}
We reviewed the scientific production on maternal health and the health of children under ten years of age, published in Journal Ciência \& Saúde Coletiva during the last 25 years, focusing on quantitative studies. The authors' characteristics, populations under study, thematic areas, and methodology are described. A total of 170 publications were identified and grouped into 12 major themes. Pregnancy, delivery, and puerperium were the subject of 47 studies, followed by child anthropometric assessments (29), breastfeeding (24), and mortality (13). The selected publications represented $3.5 \%$ of the total original papers published by the Journal since its creation in 1996 and about 5\% of the publications in the 2015-2020 period. The primary data sources were health service records, information systems, and population surveys. The cross-sectional design was used in 113 of the 170 articles, and $70 \%$ covered only one municipality. The Southeast and Northeast Regions of Brazil were the target of most studies, and the North Region was the least represented. The publications reflect the complexity of maternal and child health themes, with a particular focus on the importance of the Unified Health System and showing how open access data can contribute to public health research.

Key words Child health, Maternal health, Epidemiology, Brazil, Review
\end{abstract}

Resumo Revisamos a produção sobre saúde materna e de crianças menores de dez anos disseminada pela Revista Ciência \& Saúde Coletiva, através de revisão sistemática de publicações dos últimos 25 anos, com foco em estudos quantitativos. Características de autores e populações sob estudo, eixos temáticos e metodologia são descritos. Foram selecionadas 170 publicações classifcadas em 12 temas. Gestação, parto ou puerpério foram objeto de 47 estudos, seguidos de avaliação antropométrica (29 artigos), amamentação (24) e mortalidade (13). Os trabalhos selecionados representaram 3,5\% do total de artigos originais publicados pela Revista desde 1996, com tendência crescente, constituindo cerca de 5\% das publicações em 2015-2020. As principais fontes de dados foram registros de serviços de saúde, sistemas de informação e inquéritos populacionais. $O$ delineamento transversal foi utilizado em 113 dos 170 artigos e $70 \%$ destes abrangeram apenas um município. As Regiões Sudeste e Nordeste foram alvo da maioria dos estudos, sendo a Região Norte a menos representada. As publicações refletem a complexidade de temas que perpassam a saúde materna e da criança, com especial foco na importância do Sistema Único de Saúde e demonstrando como dados em acesso aberto podem contribuir para a pesquisa em Saúde Coletiva.

Palavras-chave Saúde da criança, Saúde materna, Epidemiologia, Brasil, Revisão 


\section{Introdução}

A Revista Ciência \& Saúde Coletiva (C\&SC) completa 25 anos destacando-se como uma das líderes no campo da saúde pública brasileira ${ }^{1}$. Essas mais de duas décadas de divulgação de estudos, resenhas e opiniões publicados pela Revista foram acompanhadas da implementação do Sistema Único de Saúde (SUS) e de importantes mudanças políticas, sociais e econômicas no Brasil².

Vivenciamos, por exemplo, expressivas mudanças no perfil epidemiológico de crianças e de mulheres que passaram pela experiência da maternidade ${ }^{2}$. Muita atenção foi dada a estas populações no que se refere a práticas de imunização, redução da mortalidade e da subnutrição, e melhoria das condições de moradia e saneamento ${ }^{2}$. Soma-se ainda o fortalecimento das evidências sobre a importância dos primeiros mil dias de vida - que incluem a gestação e os dois primeiros anos das crianças - como determinantes primordiais para a saúde dos adultos e para o desenvolvimento das populações como um todo ${ }^{3-5}$.

Apesar do progresso alcançado, alguns desafios permanecem, como as altas taxas de cesáre$a s^{6,7}$ e prematuridade ${ }^{8}$, elevada mortalidade materna ${ }^{9}$, transição da subnutrição para um cenário de sobrepeso e obesidade na infância ${ }^{10}$, aumento na ocorrência de doenças e agravos crônicos e da mortalidade por causas externas ${ }^{11}$, e pelo aparecimento de doenças transmissíveis emergentes e reemergentes ${ }^{12}$. A permanência de iniquidades, que refletem por exemplo nas condições de moradia e acesso a estruturas sanitárias adequadas, contribuem para a sustentação deste cenário². Estes exemplos apontam para a complexidade de fatores que afetam a vida de mulheres e crianças ${ }^{11}$.

Anualmente ocorrem quase três milhões de nascimentos no Brasil e a população de menores de dez anos estimada para 2020 é de 30 milhões ${ }^{13}$. Trata-se, portanto, de parcelas expressivas da população brasileira, que demandam atenção integral e focalizada, devido às suas diversas especificidades.

À luz deste histórico, o objetivo da presente revisão foi descrever as características dos estudos originais quantitativos sobre saúde materna e de crianças menores de dez anos publicados nos últimos 25 anos pela Revista C\&SC. Estudos sobre saúde da mulher, fora do escopo da saúde materna, são abordados em outro artigo no presente suplemento.

\section{Material e métodos}

\section{Estratégia de busca e seleção de estudos}

A seleção dos estudos foi realizada a partir da biblioteca eletrônica SciELO (Scientific Electronic Library Online, https://www.scielo.br/), que inclui todos os volumes da Revista, desde a sua criação em 1996. A triagem de publicações foi feita através da consulta aos sumários de todos os números publicados e disponíveis na base de dados. Para a primeira fase de seleção foi observada a pertinência dos títulos das publicações categorizadas sob os tópicos "Artigos" e "Temas Livres", desconsiderando-se, portanto, editoriais, artigos de opinião, resenhas e revisões. Em seguida os resumos foram consultados, sendo rejeitados aqueles que não atendiam aos critérios de elegibilidade descritos abaixo. Nos casos de incerteza, a publicação foi selecionada para posterior consulta ao texto completo e confirmação da adequação ao tema da revisão. As etapas de seleção foram realizadas de forma independente por duas avaliadoras (JCC e MFSM) e em caso de persistência de dúvida ou divergência, o terceiro avaliador (CGV) foi consultado.

\section{Critérios de elegibilidade}

Estudos quantitativos originais que contemplassem a população de gestantes, puérperas e crianças menores de dez anos eram elegíveis. Os temas a serem incluídos foram aqueles referentes ao pré-natal, à gestação, ao parto e ao puerpério para mulheres, e à saúde da criança em geral.

O critério de idade para a inclusão de estudos sobre a infância foi baseado na definição do Ministério da Saúde, conforme apresentado na Política Nacional de Atenção Integral à Saúde da Criança (PNAISC), de crianças como indivíduos de zero a nove anos de idade ${ }^{11}$.

Foram excluídos estudos que utilizaram métodos qualitativos, fizeram análise de políticas e programas e de qualidade de serviços de saúde tendo como unidade analítica as instituições ou os profissionais. Temas contemplados por outros artigos no presente suplemento, como saúde ambiental, saúde mental, saúde bucal, acidentes e violências, ou saúde da mulher (exceto durante a gestação, parto e puerpério), também foram excluídos. Em relação aos estudos do campo de alimentação e nutrição, foram deixados de fora 
aqueles que avaliaram consumo alimentar, mas mantidos os que analisaram o estado nutricional relacionado ao perfil de micronutrientes/biomarcadores e aos indicadores antropométricos.

\section{Extração de dados}

Das publicações selecionadas foram extraídas as seguintes informações: ano de publicação e instituição de afiliação do primeiro autor; população (mulheres ou crianças); localidade de origem da população; delineamento do estudo; origem da amostra ano do dado; desfechos; e inclusão ou não de fatores socioeconômicos.

A origem da amostra estudada foi classificada em Unidades Básicas de Saúde (UBS), hospitais/ maternidades, sistemas de informação, inquéritos populacionais, escolas, ou outras instituições.A partir da informação do artigo sobre a localidade de origem da população estudada, as publicações foram agrupadas por Unidade da Federação (UF), quando pertinente, e descritas em relação à sua abrangência, sendo caracterizadas em 'apenas um município', 'vários municípios', 'apenas um estado', 'vários estados', 'Brasil', e ‘Global' quando utilizados dados de múltiplos países.

Os estudos foram também categorizados em eixos temáticos para descrição dos conteúdos abordados. Apesar de muitos deles poderem ser classificados em mais de um grupo, optou-se pela categoria mais abrangente. Os eixos temáticos são categorias definidas por nós, autores da revisão, e utilizados para agrupamento dos estudos. São baseados nos objetivos, população-alvo, desfechos e demais conteúdos das publicações. Foram definidos para facilitar a descrição dos temas abordados.

As características dos estudos são apresentadas de forma quantitativa e na sequência é apresentada uma análise crítica dos resultados.

Uma análise bibliométrica das publicações selecionadas foi realizada consultando-se cada um dos títulos dos estudos selecionados na plataforma Google Scholar (https://scholar.google. com.br/), sendo apresentadas aquelas identificadas com maior número de citações.

\section{Resultados}

\section{Seleção dos estudos}

Na primeira etapa de busca independente foram identificados 642 artigos a partir da leitura dos títulos. Estes foram revisados por ambas as avaliadoras e 287 foram selecionados para leitura completa do texto publicado. Destes, 117 não atendiam aos critérios de elegibilidade, resultando em 170 artigos a serem incluídos para análise. Esse número corresponde a 3,5\% dos artigos originais publicados até março de 2020. O fluxo de seleção é apresentado na Figura 1.

\section{Período de publicação}

Foram identificadas publicações a partir do ano 2005 e observa-se um aumento ao longo dos anos subsequentes. Como a avaliação deste indicador por ano de publicação pode sofrer interferências de números especiais e suplementos editados pela Revista, o dado foi agrupado em períodos de cinco anos. De acordo com a Figura 2, o número de publicações selecionadas entre os períodos 2005-2009 e 2010-2014 quintuplicou, passando de 12 para 63 e mantendo-se elevado, com 95 estudos identificados entre 2015 e 2020.

Para cada um dos quinquênios apresentados o percentual de publicações cobrindo a temática de interesse em relação ao total foi, respectivamente, $1,5 \%, 3,4 \%$ e $4,7 \%$. Nestes casos foi utilizado como denominador o total de artigos publicados em cada ano, excluindo-se as categorias Editorial, Carta, Resenha e Comentário de debatedores.

Já os dados utilizados pelos autores dos estudos nas análises foram coletados entre $1990 \mathrm{e}$ 2016. Nota-se que alguns estudos falham em reportar na sessão de métodos o período a que o dado apresentado se refere.

\section{Distribuição geográfica}

Na Figura 3 estão apresentados os quantitativos de estudos por UF da (A) instituição de afiliação do primeiro autor e (B) população sob estudo. Nenhum dos estudos identificados teve autoria ou população provenientes dos estados do Amapá, Rondônia e Tocantins. Observa-se uma concentração de autoria em alguns estados das regiões Sul e Sudeste, especialmente São Paulo, Rio de Janeiro, Rio Grande do Sul e Minas Gerais. Na região Nordeste, destacam-se Pernambuco e Paraíba. Apenas 6 dos 170 (3,5\%) estudos foram conduzidos por pesquisadores vinculados a instituições da região Norte. A Fundação Oswaldo Cruz (Fiocruz) é a instituição com maior número de publicações $(\mathrm{n}=15)$, incluindo autores de diferentes estados, seguida pela Universidade Estadual da Paraíba (UEPB, n $=9$, com um mesmo autor comum a todos os 


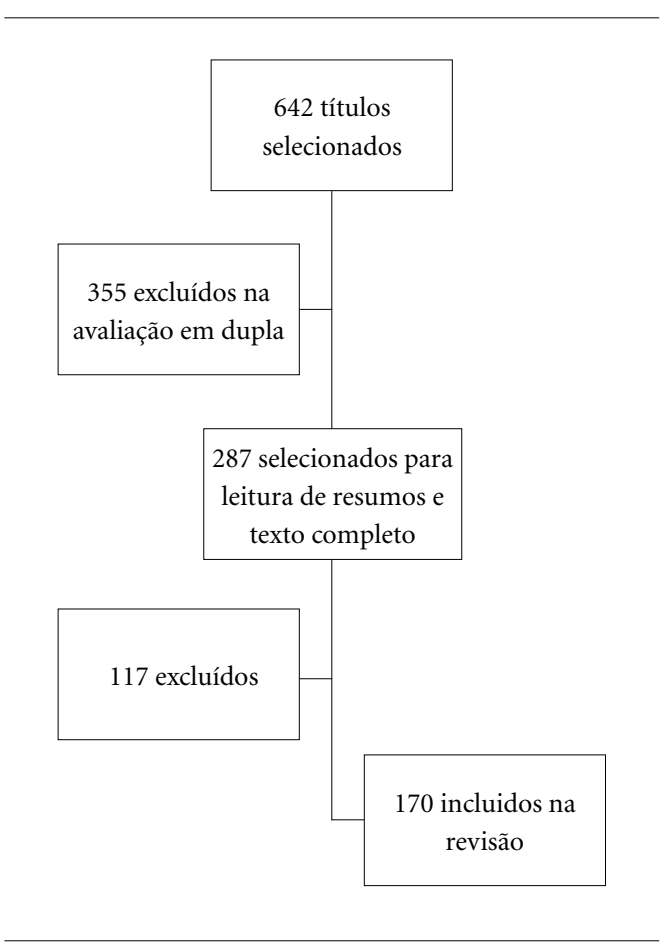

Figura 1. Processo de seleção das publicações. Revista Ciência \& Saúde Coletiva, 1996-2020. estudos selecionados) e pela Universidade Federal de Rio Grande (FURG, $\mathrm{n}=8$ ). De maneira geral, os estudos são realizados com populações da mesma UF de origem dos autores.

\section{Eixos temáticos e origem da amostra/dados}

No Quadro 1 apresentamos a classificação dos artigos em eixos temáticos, o quantitativo em cada categoria e o conteúdo que incluem.

Dos 170 estudos, 47 tinham gestantes ou puérperas como população e fazem parte do grupo temático que contempla pré-natal, parto e puerpério. Os dados para estes estudos foram majoritariamente coletados de prontuários $\mathrm{e}$ entrevistas realizadas em hospitais e maternidades; a segunda principal fonte de captação de informação são as UBS. Realização de pré-natal e gestação na adolescência são os assuntos mais abordados neste grupo.

O pré-natal foi avaliado pela perspectiva da qualidade e adequação ${ }^{14-21}$, incluindo, por exemplo, as recomendações do Programa de Humanização no Pré-natal e Nascimento ${ }^{15}$ e da articulação com as equipes do Programa de Saúde da Família (PSF) ${ }^{22}$. Desigualdades sociais ${ }^{1,23-26} \mathrm{e}$ associação da realização do pré-natal com risco reprodutivo ${ }^{27}$, peso ao nascer e prematuridade também foram avaliados pelos pesquisadores ${ }^{17,28}$.

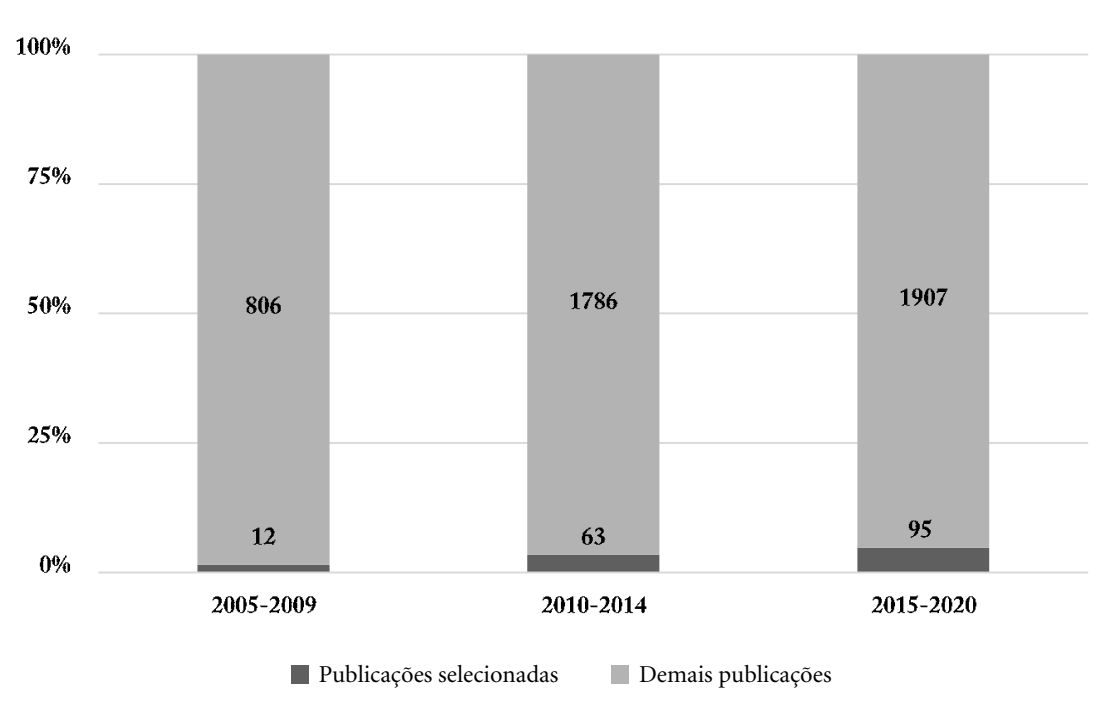

Figura 2. Quantitativo total e de publicações selecionadas na Revista Ciência \& Saúde Coletiva por quinquênio (2005-2020). 
(A) Instituição de afiliação do primeiro autor

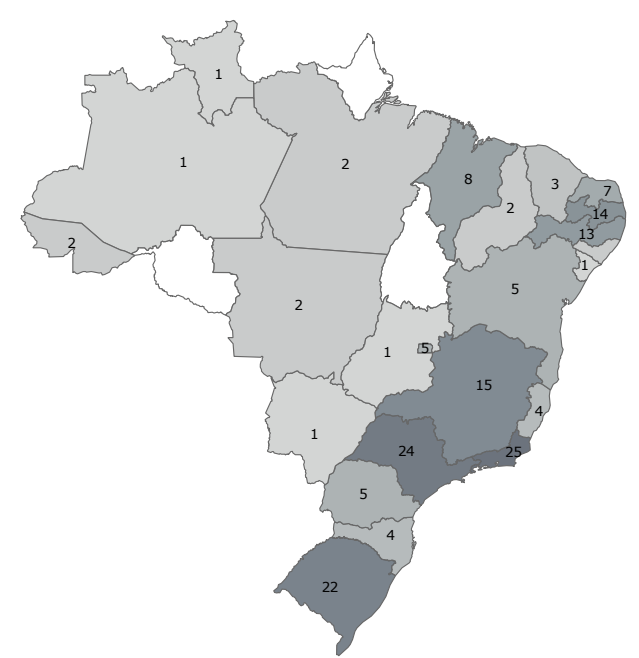

(B) Localidade de origem da população

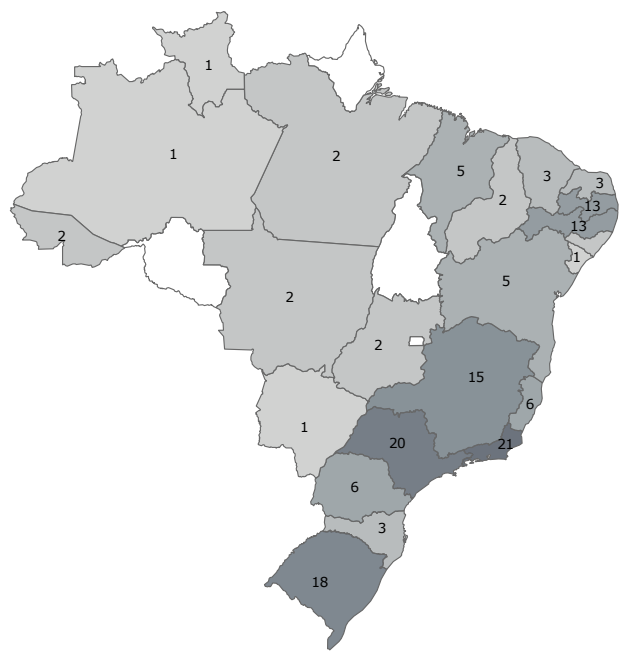

Figura 3. Distribuição geográfica da instituição de afiliação do primeiro autor e da localidade de origem da população dos estudos, por Unidade da Federação. Revista Ciência \& Saúde Coletiva, 2005-2020.

Quadro 1. Eixos temáticos, quantitativo de estudos e conteúdo das publicações incluídas na revisão. Revista Ciência \& Saúde Coletiva, artigos selecionados, 2005-2020.

\begin{tabular}{|c|c|c|}
\hline Grande tema & $\begin{array}{l}\text { Número de } \\
\text { estudos }\end{array}$ & Exemplos de conteúdo \\
\hline Gestação, parto e puerpério & 47 & $\begin{array}{l}\text { Perfil social/ sociodemográfico } \\
\text { Gravidez na adolescência } \\
\text { Escuta na assistência pré-natal } \\
\text { Definição pelo parto cesáreo } \\
\text { Cuidado na ESF } \\
\text { Morbidades relacionadas ao tipo de parto } \\
\text { Utilização de medicamentos } \\
\text { Evolução da assistência } \\
\text { Testagem HIV } \\
\text { Ganho de peso gestacional } \\
\text { Desigualdades no pré-natal } \\
\text { Near miss de morte materna } \\
\text { Conteúdo da assistência pré-natal } \\
\text { Gestação e parto na prisão } \\
\text { Dor lombar } \\
\text { Uso de álcool } \\
\text { Não realização de exame citopatológico } \\
\text { Vulnerabilidade de mulheres negras } \\
\text { Câncer de mama gestacional } \\
\text { Conhecimento e comportamento sobre toxoplasmose } \\
\text { Vacinação contra influenza }\end{array}$ \\
\hline $\begin{array}{l}\text { Estado nutricional } \\
\text { (antropometria) }\end{array}$ & 29 & $\begin{array}{l}\text { Sobrepeso/ Obesidade } \\
\text { Nanismo/ déficit de altura/ stunting } \\
\text { Percentual de gordura }\end{array}$ \\
\hline
\end{tabular}


Quadro 1. Eixos temáticos, quantitativo de estudos e conteúdo das publicações incluídas na revisão. Revista Ciência \& Saúde Coletiva, artigos selecionados, 2005-2020.

\begin{tabular}{|c|c|c|}
\hline Grande tema & $\begin{array}{c}\text { Número de } \\
\text { estudos }\end{array}$ & Exemplos de conteúdo \\
\hline Amamentação & 24 & $\begin{array}{l}\text { Duração } \\
\text { Orientações } \\
\text { Intenção de amamentar } \\
\text { Tipos de aleitamento } \\
\text { Abandono/ ausência da amamentação } \\
\text { Associação com hospitalizações }\end{array}$ \\
\hline Mortalidade & 13 & $\begin{array}{l}\text { Mortalidade neonatal } \\
\text { Mortalidade infantil } \\
\text { Mortalidade de menores de cinco anos } \\
\text { Mortalidade por AIDS } \\
\end{array}$ \\
\hline Saúde neonatal & 12 & $\begin{array}{l}\text { Método canguru } \\
\text { Baixo peso ao nascer } \\
\text { Atenção neonatal } \\
\text { Prematuridade } \\
\text { Uso de surfactantes } \\
\text { Triagem auditiva } \\
\text { Nascimentos ocorridos em hospitais SUS e não SUS }\end{array}$ \\
\hline $\begin{array}{l}\text { Estado nutricional } \\
\text { (micronutrientes/prevalência) }\end{array}$ & 9 & $\begin{array}{l}\text { Ferro } \\
\text { Retinol/ vitamina A } \\
\text { Perfil lipídico }\end{array}$ \\
\hline Uso de serviços de saúde & 9 & $\begin{array}{l}\text { Programa Saúde da Família } \\
\text { Ações de educação em saúde } \\
\text { Qualidade do cuidado na AB } \\
\text { Registro de ações na Caderneta da Criança } \\
\text { Internações/ Hospitalizações } \\
\end{array}$ \\
\hline Morbidades específicas & 7 & $\begin{array}{l}\text { Anencefalia } \\
\text { Adesão a terapia antirretroviral } \\
\text { Surto de diarreia } \\
\text { Tuberculose } \\
\text { Pneumonia } \\
\text { Microcefalia } \\
\end{array}$ \\
\hline $\begin{array}{l}\text { Características maternas e } \\
\text { desfechos na saúde da criança }\end{array}$ & 6 & $\begin{array}{l}\text { Sífilis gestacional e congênita } \\
\text { Transtornos mentais maternos e saúde da criança }\end{array}$ \\
\hline Desenvolvimento infantil & 5 & $\begin{array}{l}\text { Desenvolvimento de linguagem e motricidade } \\
\text { Relação mãe-filho } \\
\text { Desenvolvimento neuropsicomotor } \\
\text { Desenvolvimento motor e cognitivo }\end{array}$ \\
\hline Sistemas de informação & 4 & $\begin{array}{l}\text { Qualidade dos dados SINASC e SIM } \\
\text { Incompletude de declaração de óbito } \\
\text { Vigilância de óbitos fetais } \\
\text { Investigação de óbitos infantis } \\
\end{array}$ \\
\hline Outros & 5 & $\begin{array}{l}\text { Uso de medicamentos e de terapias complementares } \\
\text { Promoção e educação em saúde } \\
\text { Saúde de crianças abrigadas }\end{array}$ \\
\hline
\end{tabular}

O segundo maior foco dos estudos foi gestação na adolescência ${ }^{29-32}$, incluindo perfil socioeconômico ${ }^{33}$ e vulnerabilidade social ${ }^{34}$, corresponsabilidade paterna ${ }^{35}$ e gravidezes resultantes de estupros $^{36}$. As experiências de gravidez e aborto da população de crianças, adolescentes e jovens em situação de rua também está presente entre os temas abordados ${ }^{37}$, assim como gestação e parto em prisões ${ }^{38}$.

Em relação ao tipo de parto, foram explorados temas como a trajetória na definição pelo parto cesáreo ${ }^{39}$, fatores associados ao tipo de par- 
to $^{40}$, morbidade neonatal e maternas ${ }^{41}$ e associação com a data de nascimento ${ }^{42}$. Um único estudo explorou mortalidade de mulheres internadas para parto e aborto ${ }^{43}$ e dois avaliaram near miss de mortalidade materna ${ }^{44,45}$.

Em relação a comportamentos específicos e morbidades, foi avaliado o conhecimento e a prevenção de toxoplasmose ${ }^{46}$, fatores associados ao câncer de mama gestacional ${ }^{47}$, realização de exame citopatológico ${ }^{48}$ e teste rápido anti-HIV ${ }^{49,50}$ cobertura vacinal entre puérperas e gestantes ${ }^{51,52}$, consumo de álcool ${ }^{53}$ e medicamentos ${ }^{54}$, suplementação com ácido fólico ${ }^{55}$, e presença de dor lombar intensa ${ }^{56}$. O estado nutricional das ges$\operatorname{tantes}^{57,58}$ e sua associação com peso do bebê ao nascer e prematuridade ${ }^{59,60}$ também aparecem entre os temas abordados.

Os demais 123 estudos foram agrupados em outros 11 eixos temáticos, em que prevalecem estudos sobre estado nutricional de crianças, avaliado por antropometria, e amamentação, com 29 e 24 publicações respectivamente.

As escolas e creches foram os principais locais em que os estudos agrupados no eixo temático estado nutricional (antropometria) foram realizados. Os estudos apresentam descrição da amostra de crianças tanto para estimativas de déficit quanto para excesso dos indicadores em relação à população de referência, ou usando a média dos índices antropométricos como desfecho ${ }^{40,61-82}$. Destes, três avaliaram diferenças entre crianças beneficiárias de programas de transferência condicionada de renda ou tiveram especificamente este grupo como amostra do estudo ${ }^{62,63,71}$ e um avaliou associação com hospitalizações ${ }^{67}$. Oito publicações tiveram como foco a subnutrição em crianças $^{64,74,76-81,83}$, sendo um realizado com a população indígena Yanomami ${ }^{78}$ e outro focado em crianças da região Amazônica Acreana ${ }^{77}$. O excesso de peso foi avaliado em quatro dos estudos neste grupo temático ${ }^{73,75,82,84}$.

Além destes, uma das publicações teve como amostra crianças e adolescentes com fibrose cística e avaliou a distribuição de gordura corporal ${ }^{85}$; duas tiveram como foco diferentes métodos para análise antropométrica ${ }^{86,87}$; um avaliou ambos sobrepeso e baixa estatura em crianças nascidas com baixo peso ${ }^{88}$; e um avaliou velocidade do ganho de peso ${ }^{89}$.

Entre os 29 estudos agrupados sob o eixo temático amamentação os dados foram coletados prioritariamente em hospitais e maternidades. Padrões de amamentação ${ }^{90-96}$ e apoio ao aleitamento, através de orientações e suporte social ${ }^{97-100}$ são explorados neste eixo temático. Outros tópi- cos que aparecem com frequência são fatores associados à duração, intenção, adesão, abandono e ausência de amamentação ${ }^{101-107}$. Foram abordados também a associação com hábitos de sucção não nutritivos ${ }^{108,109}$ e com morbidades e hospitalização ${ }^{110}$; descrição de tipos de leite ofertado às crianças $^{111}$ e associação com alimentação complementar $^{112}$; e prática de aleitamento cruzado ${ }^{113}$.

Ainda na área de avaliação nutricional, dentre as nove publicações categorizadas como estado nutricional (biomarcadores/ prevalência), anemia e deficiência de vitamina A em crianças foram os desfechos mais estudados ${ }^{114-121}$ e um estudo avaliou o perfil lipídico ${ }^{122}$.

Os 12 estudos que abordam saúde neonatal avaliaram principalmente a ocorrência de baixo peso ao nascer ${ }^{123-126}$, incluindo filhos de mães adolescentes $^{125}$ e desigualdades espaciais ${ }^{124}$, e práticas de cuidado, como triagem auditiva ${ }^{127}$, uso de surfactantes ${ }^{128}$, método canguru ${ }^{129}$, e sono em decúbito dorsal ${ }^{130}$. A atenção às crianças foi também avaliada à luz do pacto para redução da mortalidade neonatal ${ }^{131}$. Características associadas à tendência temporal da prematuridade ${ }^{132} \mathrm{e} a$ qualidade de vida de nascidos pré-termo ${ }^{133}$ foram estudadas em duas publicações. A distribuição geográfica de nascimentos foi avaliada de acordo com a forma de administração da instituição (SUS ou não SUS) $)^{134}$.

Os cinco estudos que avaliaram características maternas e desfechos na saúde da criança (excluindo-se aqueles referentes à gestação e ao parto, que foram incluídos no primeiro eixo temático descrito acima), tiveram como desfecho sífilis congênita ${ }^{135-138}$ e impacto de transtorno mental, depressão e posição socioeconômica maternos na saúde da prole ${ }^{139,140}$.

$\mathrm{O}$ tema de desenvolvimento infantil - definido pelos autores como neuropsicomotor, linguagem, cognitivo - foi contemplado por cinco estudos que avaliaram fatores de risco para o desenvolvimento de crianças atendidas em $\mathrm{UBS}^{141}$, a influência do gênero $^{142}$, prejuízos na relação mãe-filho ${ }^{143}$, influência do ambiente domiciliar ${ }^{144}$ e associação com desnutrição e suplementação de vitamina $\mathrm{A}^{145}$. Todas as publicações neste grupo referem-se a crianças de até quatro anos.

Muitos estudos utilizaram dados secundários coletados de Sistemas de Informação em Saúde, especialmente aqueles disponibilizados pelo $\mathrm{Da}$ tasus, departamento de informática do Ministério da Saúde, como SIM, SINASC, SIH. Estas são as fontes utilizadas em praticamente todos os estudos agrupados no eixo temático mortalidade $(\mathrm{n}=13)$. Neste grupo estão as publicações que 
avaliaram tendência temporal ${ }^{146,147}$ e distribuição espacial $^{148-150}$ dos indicadores de mortalidade, seus determinantes ${ }^{151}$ e fatores de risco ${ }^{152,153}$. Os autores exploraram também desigualdades ${ }^{154} \mathrm{e}$ evitabilidade de mortes ${ }^{155}$, incluindo análise de acordo com raça/cor da pele ${ }^{156} \mathrm{e}$ impacto das crises econômicas na mortalidade ${ }^{157}$. Os estudos foram, em sua maioria, sobre mortalidade infantil. Dois exploraram mortalidade neonatal ${ }^{150,152}$, um estudou crianças menores de cinco anos ${ }^{157}$ - incluindo mortalidade infantil, neonatal e de zero a quatro anos - e um estudo avaliou mortalidade por Aids em crianças e adolescentes ${ }^{158}$.

Além dos estudos sobre os indicadores de mortalidade, aqueles incluídos no grupo sistemas de informação, que incluíam também as fichas de vigilância de óbitos foram utilizados em publicações cujo objetivo foi avaliar fatores associados à incompletude dos sistemas e à qualidade dos dados $^{159-162}$.

A categoria temática morbidades específicas incluiu sete estudos com os seguintes desfechos: adesão à terapia antirretroviral ${ }^{163}$, diarreia ${ }^{16,165}$, tuberculose $^{166}$, pneumonia ${ }^{167}$, microcefalia ${ }^{168} \mathrm{e}$ anencefalia ${ }^{169}$.

Estudos agrupados sob o eixo temático uso de serviços de saúde abordaram associação das condições de saúde ${ }^{170}$ e utilização de serviço pelas crianças com o PSF ${ }^{171}$ e Atenção Básica ${ }^{172}$; uso da Caderneta de Saúde da Criança ${ }^{173,174}$; internações por condições crônicas complexas ${ }^{175,176}$ e hospitalização por asma ${ }^{177}$ e diarreia ${ }^{178}$.

$\mathrm{Na}$ categoria de eixos temáticos denominada outros foram incluídos estudos sobre uso de medicamentos $^{179}$ e terapias complementares ${ }^{180}$ por crianças, situação de saúde de crianças institucionalizadas ${ }^{181}$, estudo sobre educação em saú$\mathrm{de}^{182}$ e avaliação de impacto de um programa de promoção da saúde ${ }^{183}$.

Na Figura 4, são apresentados os gráficos que sintetizam as características metodológicas dos 170 estudos: a) abrangência, b) delineamento, c) origem da amostra/dados, d) presença de fatores socioeconômicos.

\section{Abrangência}

Aproximadamente $70 \%$ das publicações abrangem apenas um município $(\mathrm{n}=115)$; as demais se dividem de maneira equilibrada entre abrangência estadual $(n=20)$, nacional $(n=15)$, ou envolvendo mais de um município $(\mathrm{n}=16)$; apenas uma publicação utilizou dados de múltiplos países em um estudo ecológico sobre mortalidade na infância (Figura 4a).

\section{Delineamento}

O delineamento transversal predomina ( $\mathrm{n}$ =132), sendo três destes aninhados a coortes e um a estudo de caso-controle. Os demais delineamentos foram os estudos ecológicos e as séries temporais, usados em 20 publicações, as coortes/ estudos longitudinais, identificados em 11 estudos, e os estudos de caso-controle, presentes em 7 publicações (Figura $4 b$ ).

\section{Origem da amostra/dados}

Serviços de saúde são a principal fonte das amostras usadas nos estudos selecionados. Juntos, UBS, hospitais e maternidades foram a base amostral de 77 estudos (45\%) (Figura 4c). Além dos sistemas de informação já citados, usados em $35(20 \%)$ estudos, foram identificadas 29 (17\%) publicações que utilizaram dados provenientes de inquéritos populacionais. A análise de inquéritos utilizou dados das seguintes fontes: Pesquisa Nacional de Demografia e Saúde (PNDS), realizada em 2006; Pesquisa Nacional por Amostra de Domicílios (PNAD), de 1993 a 2008; Pesquisa Estadual de Saúde e Nutrição (PESN) II e III, realizadas respectivamente em 1997 e 2006 no estado de Pernambuco; Chamada Neonatal: avaliação da atenção ao pré-natal e aos menores de um ano nas regiões Norte e Nordeste de 20132014; Estudo Nacional de Fortificação caseira da Alimentação Complementar (ENFAC); Fatores etiológicos do nascimento pré-termo e consequências dos fatores perinatais na saúde da criança: Coortes de nascimentos em duas cidades brasileiras - BRISA; Pesquisa de Orçamento Familiar (POF) 2008-2009; Pesquisa Nacional de Saúde de 2013; e um inquérito domiciliar realizado em Fortaleza, capital do Ceará, e outros municípios rurais. Além destes, as escolas foram locais de origem das amostras em 22 (13\%) estudos e outras instituições em 7 (4\%) deles.

\section{Fatores socioeconômicos}

Variáveis relacionadas à posição socioeconômica estavam presentes em 129 (76\%) das publicações (Figura 4d). Foram considerados para esta caracterização aqueles que incluíram renda, riqueza ou escolaridade, em estudos com dados individuais e abastecimento de água, e um índice de desenvolvimento municipal em estudos ecológicos. 


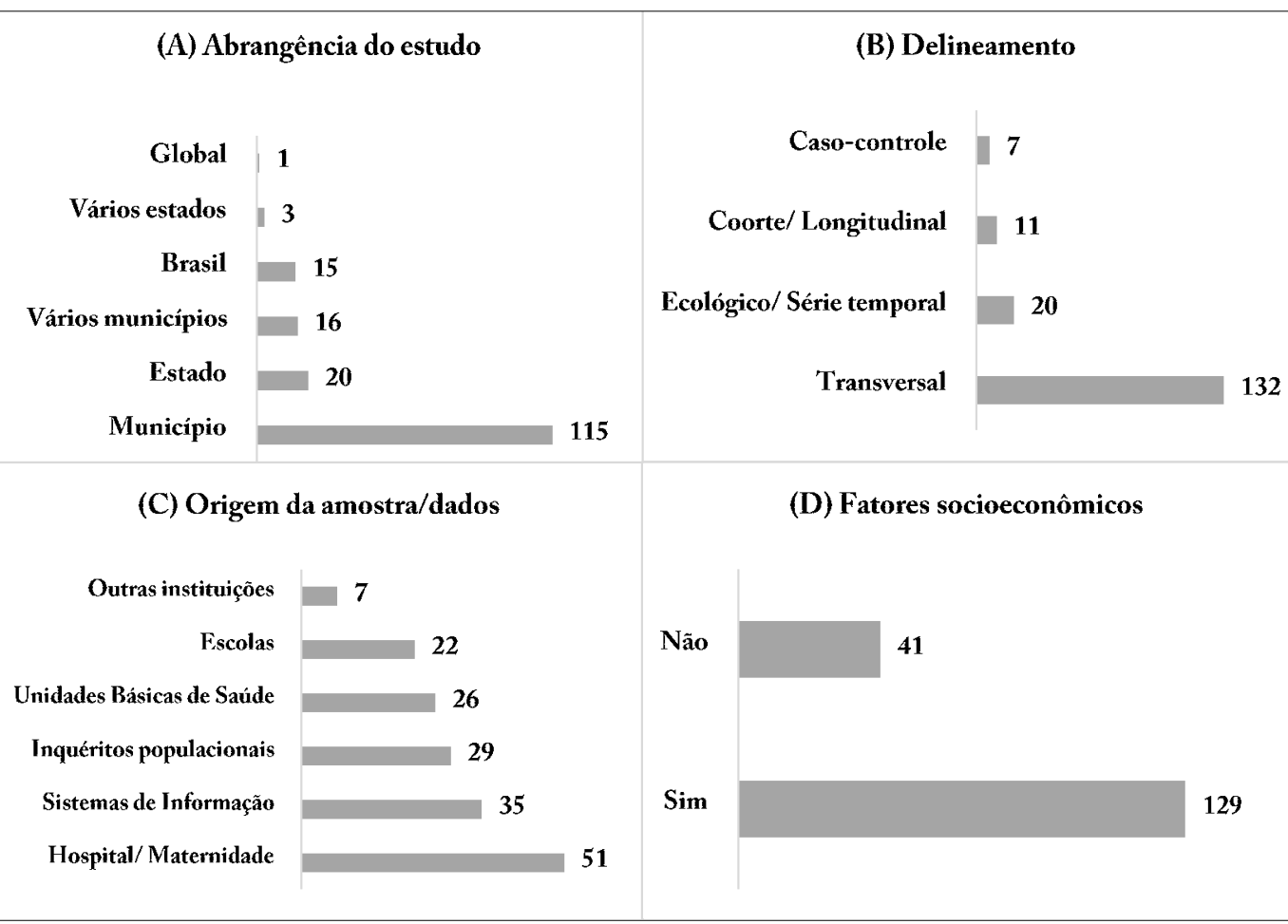

Figura 4. Número de publicações incluídas na revisão de acordo com características dos dados (A) Abrangência do estudo, (B) Delineamento, (C) Origem da amostra/dados, (D) Fatores socioeconômicos. Revista Ciência \& Saúde Coletiva, artigos selecionados, 2005-2020.

\section{Citações}

A análise bibliométrica realizada para avaliar os artigos mais citados dentre os 170 selecionados, identificou três deles com mais de 100 citações. Os temas abordados foram o impacto do PSF sobre indicadores de saúde da criança em municípios do Nordeste, com 133 citações e publicado em $2006^{170}$; a trajetória das mulheres na definição pelo parto cesáreo no Rio de Janeiro, com 149 , de $2008^{39}$; e avaliação do cuidado à saúde da gestante no contexto do PSF, de $2009^{22}$.

\section{Discussão}

A Revista C\&SC é parte fundamental do processo de institucionalização da pesquisa sobre Saúde Coletiva no Brasil ${ }^{184}$. Estudos sobre a infância ganharam espaço a partir do ano 2005 e tiveram um importante aumento ao longo do tempo. Trata-se de um período em que observamos nacionalmente a redução das taxas de mortalidade por causas evitáveis, das prevalências de doenças infecciosas e subnutrição, dentre outros fatores que impactam na sobrevivência e no pleno desenvolvimento de crianças ${ }^{2}$.

Durante este período, ocorreu em nosso país a implantação de diversas ações, programas e políticas públicas com foco na melhoria das condições de saúde da população, tanto de gestantes e puérperas, como de crianças. São alguns exemplos de inciativas nacionais: Política Nacional de Atenção Obstétrica e Neonatal (2005) ${ }^{185}$, Rede Cegonha (2011) $)^{186}$, Política Nacional de Atenção Integral à Saúde da Criança $(2015)^{187}$, Política Nacional de Alimentação e Nutrição (2012) ${ }^{188}$, Guia alimentar para a população Brasileira $(2014)^{189}$, Política Nacional de Atenção Básica (2012) ${ }^{190}$ e os diversos Cadernos de Atenção Básica temáti$\cos ^{191,192}$. A Revista cumpriu um papel importante de contribuir para a avaliação de vários destes programas, direta ou indiretamente.

Apesar do aumento no número de publicações ao longo dos anos, tanto os autores quanto as amostras estudadas ainda estão concentradas em estados das regiões Sul e Sudeste do país. Mesmo no Nordeste, que apresenta números im- 
portantes de artigos, há concentração dos estudos em apenas dois estados. Este fenômeno não é exclusivo do campo da Saúde Coletiva, e a maior parte das publicações brasileiras, em todas as áreas, são de autores vinculados a instituições da região Sudeste ${ }^{193}$. Vale ressaltar que os periódicos mais citados da área são vinculados a instituições desta região, o que pode funcionar como elemento impulsionador para autores locais ${ }^{194}$.

Em relação à abrangência dos estudos, observa-se que são majoritariamente realizados com amostras de um único município. Estudos de abrangência local apresentam fundamental importância no que tange às análises de desigualdades em saúde, tão presentes no país. Ainda que nacionalmente o Brasil tenha apresentado grandes melhorias em saúde da mulher e da criança, como citado anteriormente, nem todas as regiões e estados apresentaram a mesma tendência ou evoluíram no mesmo compasso ${ }^{195}$. Desigualdades regionais foram reportadas em diversos estudos incluídos na revisão, especialmente através de análises espaciais ${ }^{124,134,138,148-150,165,166}$. Evidências de desigualdades persistentes entre as regiões do país, apesar de estarem reduzindo para muitos indicadores, foram encontradas em análise temporal das condições de saúde da mulher e da criança ${ }^{195}$.

Avaliar subgrupos da população e ter um olhar mais próximo das demandas locais é uma forma de compreender as especificidades e instrumentalizar a tomada de decisões, o que possibilita a formulação de políticas públicas de forma a diminuir iniquidades.

A amostra de publicações selecionadas reflete a diversidade de temas que perpassam o campo da saúde materna e infantil. Observa-se, entretanto, uma centralização de estudos no campo de alimentação e nutrição, especialmente em relação ao estado nutricional de crianças, avaliado por indicadores antropométricos e com especial foco no crescimento linear, deficiência de micronutrientes e práticas de amamentação. Além deste grupo temático, destacam-se os estudos sobre mortalidade, importante indicador da qualidade de vida e de cuidado das crianças menores de cinco anos.

Em relação às fontes de dados, os sistemas de informação nacionais apresentam-se como importantes ferramentas para a análise da situação de saúde de mulheres e crianças no Brasil, com destaque para o Sistema de Informações sobre Nascidos Vivos (SINASC) e o Sistema de Informação de Mortalidade (SIM). Ainda, apesar de menos frequentes, o Sistema de Internações
Hospitalares (SIH) e até mesmo o censo populacional figuram como fontes de dados para os estudos analisados. O uso de dados secundários, coletados rotineiramente nos serviços de saúde, é essencial para aprimorar a qualidade dos sistemas de informação. Apesar desta importância, alguns estudos relatam a baixa confiabilidade dos sistemas de informação, que pode ocorrer por não preenchimento de campos básicos nos documentos originais ou durante a inclusão no próprio sistema, características que também dependem das condições estruturais de cada localidade ${ }^{161}$. Ainda assim, de maneira geral, observa-se melhoria dos sistemas nacionais, tanto em relação à cobertura, quanto à qualidade dos dados $^{196}$.

Além de dados coletados de forma sistemática pelo SUS, os inquéritos e as pesquisas com abrangência local ou nacional apareceram expressivamente nos estudos identificados. Por outro lado, o inquérito nacional mais recente, especificamente sobre saúde materno-infantil, a PNDS, foi realizada em 2006, o que dificulta estudos sobre análises de tendências de base populacional. Prontuários foram fontes frequentes de dados para os estudos, assim como informações primárias coletadas em escolas e UBS.

Avanços que ocorreram no SUS, no que diz respeito à atenção à saúde, também se refletem nas publicações, com os equipamentos de Atenção Básica e a Estratégia de Saúde da Família aparecendo em destaque. Diversos estudos incluídos nesta revisão utilizam informações disponíveis nas UBS, como os prontuários e a Caderneta de Saúde da criança, que podem ser importantes impulsionadores da articulação de pesquisadores com equipes que atuam na rede de atenção à saúde, identificando lacunas e propondo estratégias para melhorar a qualidade e a cobertura das informações ${ }^{173,174}$. Registros dos serviços de saúde são importantes fontes de dados subnacionais, por município, região ou distrito, relacionadas às decisões de gestão e à dinâmica local, funcionando também como instrumentos de avaliação da qualidade do serviço ${ }^{197}$.

A diversidade de fontes utilizadas nos artigos selecionados revela a importância da articulação entre as três esferas de gestão do SUS, além da articulação intersetorial, especialmente com a área da Educação.

Análises de desigualdades socioeconômicas e regionais fazem parte essencial dos estudos de Saúde Coletiva no Brasil, uma vez que estas exercem forte influência sobre condições de vida e de saúde, sendo refletidas na cobertura de in- 
tervenções, no estado nutricional e em outros indicadores ${ }^{2}$. A maioria dos artigos publicados reportam variáveis socioeconômicas, evidenciando a importância de se levar em consideração estes aspectos na descrição da amostra, na análise dos dados e na interpretação dos resultados.

Ao comparar a temática das publicações analisadas, observa-se que a Revista tem abordado sistematicamente a grande maioria dos problemas de saúde que afetam gestantes, puérperas e crianças brasileiras. Nota-se, no entanto, que temas como mortalidade materna, prematuridade, desenvolvimento psicomotor e obesidade infantil foram alvos de menos artigos do que seria esperado em função de sua importância no contexto brasileiro.

Em síntese, diante do balanço da trajetória da Revista C\&SC, podemos afirmar que alguns dos principais elementos dessa história de 25 anos são a diversidade de temas abordados pelos autores sobre elementos que impactam a vida de mulheres e crianças e que permitem uma visão ampla das ações que ocorrem no âmbito do SUS, especialmente no nível municipal; a multiplicidade de instituições que submetem sua produção científica para este periódico; e a possibilidade acesso a todos os volumes, de forma organizada, no SciELO, com acesso aberto e gratuito.

Alguns desafios se apresentam para os próximos anos da revista e se misturam com os desafios da ciência no Brasil, como apoio à descentralização da produção e avanços na internacionalização, projeto já em andamento com a possibilidade de publicação em dois idiomas.

O presente artigo de revisão apresenta o ponto forte de incluir estudos sobre gestação, parto e puerpério, períodos de grande impacto para a saúde das mulheres, mas também determinantes para o desenvolvimento de crianças, especialmente nos dois primeiros anos de vida. Além disso, a triagem de artigos pelo sumário de todos os números publicados pela Revista C\&SC permitiu a identificação mais precisa dos estudos a serem incluídos.

Podem ser consideradas como limitações da revisão a não inclusão de estudos avaliados em outros artigos do suplemento (como saúde bucal, saúde mental, saúde ambiental e acidentes e violências), decisão tomada com o intuito de evitar a duplicidade na descrição das publicações. Esse critério de exclusão reduz o número de estudos reportados neste trabalho por não contemplar a totalidade daqueles realizados com a população menor de dez anos, restringindo sua abrangência.

\section{Conclusão}

A experiência do Brasil tem apontado sucesso de intervenções voltadas para atenção à gestação, nascimento e infância e a Revista C\&SC tem acompanhado esta trajetória. Diversos são os marcos históricos que se confundem e influenciam a história da Revista e o perfil dos estudos publicados tende a acompanhar estes processos.

As publicações científicas são um importante componente da prática na saúde coletiva, e o conjunto de estudos publicados pela Revista C\&SC contempla um rico material de pesquisa, refletindo experiências ocorridas no âmbito do SUS e a partir da articulação intersetorial e interfederativa, tão caras à promoção da saúde.

\section{Colaboradores}

JC Costa e MFS Maia conduziram o processo de triagem e seleção dos artigos, extração de dados e análise. JC Costa, MFS Maia e CG Victora participaram da concepção do trabalho, interpretação dos dados e redação do manuscrito e aprovaram a versão final. 


\section{Referências}

1. Minayo MCS, Gomes R. Cien Saude Colet no contexto nacional e internacional da divulgação científica. Cien Saude Colet 2015; 20(7):2013-2022.

2. Victora CG, Aquino EML, Leal MC, Monteiro CA, Barros FC, Szwarcwald CL. Maternal and child health in Brazil: progress and challenges. Lancet 2011; 377(9780):1863-1876.

3. Adair LS, Fall CHD, Osmond C, Stein AD, Martorell R, Ramirez-Zea M, Sachdev HS, Dahly DL, Bas I, Norris SA, Micklesfield L, Hallal P, Victora CG. Associations of linear growth and relative weight gain during early life with adult health and human capital in countries of low and middle income: findings from five birth cohort studies. Lancet 2013; 382(9891):525-534.

4. Martorell R, Horta BL, Adair LS, Stein AD, Richter L, Fall CHD, Bhargava SK, Biswas SKD, Perez L, Barros FC, Victora CG, Consortium on Health Orientated Research in Transitional Societies G. Weight gain in the first two years of life is an important predictor of schooling outcomes in pooled analyses from five birth cohorts from low- and middle-income countries. $J$ Nutr 2010; 140(2):348-354.

5. Victora CG, Horta BL, Mola CL, Quevedo L, Pinheiro RT, Gigante DP, Gonçalves H, Barros FC. Association between breastfeeding and intelligence, educational attainment, and income at 30 years of age: a prospective birth cohort study from Brazil. Lancet Glob Health 2015; 3(4):e199-e205.

6. Barros AJD, Victora CG, Horta BL, Wehrmeister FC, Bassani D, Silveira MF, Santos LP, Blumenberg C, Barros FC. Antenatal care and caesarean sections: trends and inequalities in four population-based birth cohorts in Pelotas, Brazil, 1982-2015. Int J Epidemiol 2019; 48(Supl. 1):i37-i45.

7. Barros AJD, Santos IS, Matijasevich A, Domingues MR, Silveira M, Barros FC, Victora CG. Patterns of deliveries in a Brazilian birth cohort: almost universal cesarean sections for the better-off. Rev Saude Publica 2011; 45(4):635-643.

8. Silveira MF, Santos IS, Barros AJD, Matijasevich A, Barros FC, Victora CG. Aumento da prematuridade no Brasil: revisão de estudos de base populacional. Rev Saude Publica 2008; 42(5):957-964.

9. Silva BG, Lima NP, Silva SG, Antunez SF, Seerig LM, Restrepo-Mendez MC, Wehrmeister FC. Maternal mortality in Brazil from 2001 to 2012: time trends and regional differences. Rev Bras Epidemiol 2016; 19(3):484-493.

10. Coutinho JG, Gentil PC, Toral N. A desnutrição e obesidade no Brasil: o enfrentamento com base na agenda única da nutrição. Cad Saude Publica 2008; 24(Supl. 2):S332-S340.

11. Brasil. Ministério da Saúde (2018). Política Nacional de Atenção Integral à Saúde da Criança: orientações para implementação. Brasília: MS; 2018.

12. Waldman EA, Sato AP. Path of infectious diseases in Brazil in the last 50 years: an ongoing challenge. Rev Saude Publica 2016; 50:68.

13. Instituto Brasileiro de Geografia e Estatística (IBGE). Estatísticas do Registro Civil: nascidos vivos, por ano de nascimento (Tabela 2679). Rio de Janeiro: IBGE; 2018.
14. Niquini RP, Bittencourt SA, Lacerda EM, Saunders C, Leal MC. Avaliação do processo da assistência nutricional no pré-natal em sete unidades de saúde da família do Município do Rio de Janeiro. Cien Saude Colet 2012; 17(10):2805-2816.

15. Mendes RB, Santos JMJ, Prado DS, Gurgel RQ, Bezerra FD, Gurgel RQ. Avaliação da qualidade do prénatal a partir das recomendações do Programa de Humanização no Pré-natal e Nascimento. Cien Saude Colet 2020; 25(3):793-804.

16. Martinelli KG, Santos Neto ETD, Gama SGN, Oliveira AE. Acesso ao pré-natal: desigualdades em região de alta mortalidade materna do sudeste brasileiro. Cien Saude Colet 2016; 21(5):1647-1658.

17. Gonzaga IC, Santos SL, Silva AR, Campelo V. Atenção pré-natal e fatores de risco associados à prematuridade e baixo peso ao nascer em capital do nordeste brasileiro. Cien Saude Colet 2016; 21(6):1965-1974.

18. Santos LAV, Lara MO, Lima RCR, Rocha AF, Rocha EM, Gloria JCR, Ribeiro GC. História gestacional e características da assistência pré-natal de puérperas adolescentes e adultas em uma maternidade do interior de Minas Gerais, Brasil. Cien Saude Colet 2018; 23(2):617-625.

19. Goudard MJ, Simoes VM, Batista RF, Queiroz RC, Alves MT, Coimbra LC, Martins MG, Barbieri MA, Nathasje IF. Inadequação do conteúdo da assistência prénatal e fatores associados em uma coorte no nordeste brasileiro. Cien Saude Colet 2016; 21(4):1227-1238.

20. Durães-Pereira MB, Novo NF, Armond JE. A escuta e o diálogo na assistência ao pré-natal, na periferia da zona Sul, no município de São Paulo. Cien Saude Colet 2007; 12(2):465-476.

21. Mario DN, Rigo L, Boclin KLS, Malvestio LMM, Anziliero D, Horta BL, Wehrmeister FC, Martinez-Mesa J. Qualidade do pré-natal no Brasil: Pesquisa Nacional de Saude 2013. Cien Saude Colet 2019; 24(3):12231232.

22. Costa GD, Cotta RM, Reis JR, Siqueira-Batista R, Gomes AP, Franceschini SC. Avaliação do cuidado a saúde da gestante no contexto do Programa Saude da Família. Cien Saude Colet 2009; 14(Supl. 1):1347-1357.

23. Fonseca SC, S MD, Pereira CM, Scoralick AC, Jorge MG, Rozario S. Desigualdades no pré-natal em cidade do Sudeste do Brasil. Cien Saude Colet 2014; 19(7):1991-1998.

24. Garcia EM, Martinelli KG, Gama SGND, Oliveira AE, Esposti CDD, Santos Neto ETD. Risco gestacional e desigualdades sociais: uma relacao possivel? Cien Saude Colet 2019; 24(12):4633-4642.

25. Theophilo RL, Rattner D, Pereira EL. Vulnerabilidade de mulheres negras na atenção ao pré-natal e ao parto no SUS: análise da pesquisa da Ouvidoria Ativa. Cien Saude Colet 2018; 23(11):3505-3516.

26. Pinto LF, Malafaia MF, Borges JA, Baccaro A, Soranz DR. Perfil social das gestantes em unidades de saúde da família do município de Teresópolis. Cien Saude Colet 2005; 10(1):205-213.

27. Xavier RB, Jannotti CB, Silva KS, Martins AC. Risco reprodutivo e renda familiar: análise do perfil de gestantes. Cien Saude Colet 2013; 18(4):1161-1171. 
28. Noronha GA, C LM, Lira PI, Veras AA, Gonçalves FC, Batista Filho M. Evolução da assistência materno-infantil e do peso ao nascer no Estado de Pernambuco em 1997 e 2006. Cien Saude Colet 2012; 17(10):27492756.

29. Béria JU, Schermann LB, Leal AF, Hilgert JB, Stein AT, Alves GG, Câmara S, Palazzo L. Maternidade na adolescência inicial: estudo caso-controle no sul do Brasil. Cien Saude Colet 2020; 25(2):439-448.

30. Rossetto MS, Schermann LB, Beria JU. Maternidade na adolescência: indicadores emocionais negativos e fatores associados em mães de 14 a 16 anos em Porto Alegre, RS, Brasil. Cien Saude Colet 2014; 19(10):4235-4246.

31. Santos MM, Barros DC, Baiao MR, Saunders C. Atenção nutricional e ganho de peso gestacional em adolescentes: uma abordagem quantiqualitativa. Cien Saude Colet 2013; 18(3):789-802.

32. Santos NL, Costa MC, Amaral MT, Vieira GO, Bacelar EB, Almeida AH. Gravidez na adolescência: análise de fatores de risco para baixo peso, prematuridade e cesariana. Cien Saude Colet 2014; 19(3):719-726.

33. Paraguassú ALCB, Costa MCO, Nascimento Sobrinho CL, Patel BN, Freitas JT, Araújo FPO. Situação sociodemográfica e de saúde reprodutiva pré e pós-gestacional de adolescentes, Feira de Santana, Bahia, Brasil. Cien Saude Colet 2005; 10(2):373-380.

34. Silva KS, Rozenberg R, Bonan C, Chuva VC, Costa SF, Gomes MA. Gravidez recorrente na adolescencia e vulnerabilidade social no Rio de Janeiro (RJ, Brasil):uma análise de dados do Sistema de Nascidos Vivos. Cien Saude Colet 2011; 16(5):2485-2493.

35. Costa MCO, Lima IC, Martins Júnior DF, Santos CAST, Araújo FPO, Assis DR. Gravidez na adolescência e co-responsabilidade paterna: trajetória sociodemográfica e atitudes com a gestação e a criança. Cien Saude Colet 2005; 10(3):719-727.

36. Souto RMCV, Porto DL, Pinto IV, Vidotti CCF, Barufaldi LA, Freitas MG, Silva MMAD, Lima CM. Estupro e gravidez de meninas de até 13 anos no Brasil: características e implicações na saúde gestacional, parto e nascimento. Cien Saude Colet 2017; 22(9):2909-2918.

37. Neiva-Silva L, Demenech LM, Moreira LR, Oliveira AT, Carvalho FT, Paludo SDS. Experiência de gravidez e aborto em crianças, adolescentes e jovens em situação de rua. Cien Saude Colet 2018; 23(4):1055-1066.

38. Leal MC, Ayres BVS, Esteves-Pereira AP, Sánchez AR, Larouzé B. Nascer na prisão: gestação e parto atrás das grades no Brasil. Cien Saude Colet 2016; 21(7):20612070.

39. Dias MA, Domingues RM, Pereira AP, Fonseca SC, Gama SG, Theme Filha MM, Bittencourt SD, Rocha PM, Schilithz AO, Leal MC. Trajetória das mulheres na definição pelo parto cesáreo: estudo de caso em duas unidades do sistema de saúde suplementar do estado do Rio de Janeiro. Cien Saude Colet 2008; 13(5):1521-1534.

40. Meller FO, Schafer AA. Fatores associados ao tipo de parto em mulheres brasileiras: PNDS 2006. Cien Saude Colet 2011; 16(9):3829-3835.
41. Cardoso PO, Alberti LR, Petroianu A. Morbidade neonatal e maternas relacionada ao tipo de parto. Cien Saude Colet 2010; 15(2):427-435.

42. Chiavegatto Filho AD. Partos cesáreos e a escolha da data de nascimento no Município de São Paulo. Cien Saude Colet 2013; 18(8):2413-2420.

43. Kale PL, Jorge MHPM, Fonseca SC, Cascao AM, Silva KSD, Reis AC, Taniguchi MT. Mortes de mulheres internadas para parto e por aborto e de seus conceptos em maternidades públicas. Cien Saude Colet 2018; 23(5):1577-1590.

44. Rosendo TM, Roncalli AG. Near miss materno e iniquidades em saúde: análise de determinantes contextuais no Rio Grande do Norte, Brasil. Cien Saude Colet 2016; 21(1):191-201.

45. Rosendo TM, Roncalli AG. Prevalência e fatores associados ao Near Miss Materno: inquérito populacional em uma capital do Nordeste Brasileiro. Cien Saude Colet 2015; 20(4):1295-304.

46. Moura IPDS, Ferreira IP, Pontes AN, Bichara CNC. Conhecimento e comportamento preventivo de gestantes sobre Toxoplasmose no município de Imperatriz, Maranhão, Brasil. Cien Saude Colet 2019; 24(10):3933-3946.

47. Monteiro DLM, Nunes CL, Rodrigues NCP, Antunes CA, Almeida EM, Barmpas DBS, Magalhães ALC, Trajano AJB. Fatores associados ao câncer de mama gestacional: estudo caso-controle. Cien Saude Colet 2019; 24(6):2361-2369.

48. Terlan RJ, Cesar JA. Não realização de citopatológico de colo uterino entre gestantes no extremo sul do Brasil: prevalência e fatores associados. Cien Saude Colet 2018; 23(11):3557-3566.

49. Soares ML, Oliveira MI, Fonseca VM, Brito AS, Silva KS. Preditores do desconhecimento do status sorológico de HIV entre puérperas submetidas ao teste rápido anti-HIV na internação para o parto. Cien Saude Colet 2013; 18(5):1313-1320.

50. Oliveira MIC, Silva KSD, Gomes DM. Fatores associados a submissão ao teste rápido anti-HIV na assistência ao parto. Cien Saude Colet 2018; 23(2):575-584.

51. Rocha BC, Carvalheira AP, Ferrari AP, Tonete VL Duarte MT, Parada CM. Cobertura vacinal e fatores associados em puérperas de município paulista. Cien Saude Colet 2016; 21(7):2287-2292.

52. Mendoza-Sassi RA, Linhares AO, Schroeder FMM, Maas NM, Nomiyama S, César JA. Vacinação contra Influenza entre gestantes no Sul do Brasil e fatores associados. Cien Saude Colet 2019; 24(12):4655-4664.

53. Guimaraes VA, Fernandes KS, Lucchese R, Vera I, Martins BCT, Amorim TA, Guimaraes RA. Prevalência e fatores associados ao uso de álcool durante a gestação em uma maternidade de Goiás, Brasil Central. Cien Saude Colet 2018; 23(10):3413-3420.

54. Brum LF, Pereira P, Felicetti LL, Silveira RD. Utilização de medicamentos por gestantes usuárias do Sistema Único de Saúde no município de Santa Rosa (RS, Brasil). Cien Saude Colet 2011; 16(5):2435-2442.

55. Linhares AO, Cesar JA. Suplementação com ácido fólico entre gestantes no extremo Sul do Brasil: prevalência e fatores associados. Cien Saude Colet 2017; 22(2):535-542. 
56. Duarte VM, Meucci RD, Cesar JA. Dor lombar intensa em gestantes do extremo Sul do Brasil. Cien Saude Colet 2018; 23(8):2487-2494.

57. Santos EN, Velarde LG, Ferreira VA. Associação entre deficiência de vitamina A e variáveis socioeconômicas, nutricionais e obstétricas de gestantes. Cien Saude Colet 2010; 15(Supl. 1):1021-1030.

58. Ferreira LB, Melo LF, Melo MEF, Sousa TM, Miranda C, Pereira SCL, Notaro KAM, Santos LCD. Fatores assistenciais e gestacionais associados a anemia em nutrizes atendidas em um banco de leite humano. Cien Saude Colet 2018; 23(11):3567-3575.

59. Fonseca MR, Laurenti R, Marin CR, Traldi MC. Ganho de peso gestacional e peso ao nascer do concepto: estudo transversal na região de Jundiaí, São Paulo, Brasil. Cien Saude Colet 2014; 19(5):1401-1407.

60. Oliveira ACM, Pereira LA, Ferreira RC, Clemente APG. Estado nutricional materno e sua associação com o peso ao nascer em gestações de alto risco. Cien Saude Colet 2018; 23(7):2373-2382.

61. Martino HS, Ferreira AC, Pereira CN, Silva RR. Avaliação antropométrica e análise dietética de pré-escolares em centros educacionais municipais no sul de Minas Gerais. Cien Saude Colet 2010; 15(2):551-558.

62. Monteiro F, Schmidt ST, Costa IB, Almeida CC, Matuda NS. Bolsa Família: insegurança alimentar e nutricional de crianças menores de cinco anos. Cien Saude Colet 2014; 19(5):1347-1357.

63. Vega JB, Taddei JA, Poblacion AP. Características sociodemográficas e nutricionais de crianças brasileiras menores de 2 anos beneficiárias de programas de transferência condicionada de renda em 2006. Cien Saude Colet 2014; 19(3):931-942.

64. Souza MM, Pedraza DF, Menezes TN. Estado nutricional de crianças assistidas em creches e situação de (in)seguranca alimentar de suas famílias. Cien Saude Colet 2012; 17(12):3425-3436.

65. Pereira LFDS, Andrade LMB, Spyrides MHC, Lyra CO. Estado nutricional de menores de 5 anos de idade no Brasil: evidências da polarização epidemiológica nutricional. Cien Saude Colet 2017; 22(10):3341-3352.

66. Pedraza DF, Silva FA, Melo NLS, Araujo EMN, Sousa CPC. Estado nutricional e hábitos alimentares de escolares de Campina Grande, Paraíba, Brasil. Cien Saude Colet 2017; 22(2):469-477.

67. Pedraza DF. Hospitalização por doenças infecciosas, parasitismo e evolução nutricional de crianças atendidas em creches públicas. Cien Saude Colet 2017; 22(12):4105-4114.

68. Pedraza DF, Oliveira MM, Rocha ACD, Araújo EMN. Índices antropométricos de crianças assistidas em creches e sua relação com fatores socioeconômicos, maternos e infantis. Cien Saude Colet 2016; 21(7):22192232.

69. Lourenco AEP, Vieira JL, Rocha CMMD, Lima FF. Influência da ambiência escolar no estado nutricional de pré-escolares de Macaé, Rio de Janeiro, Brasil. Cien Saude Colet 2019; 24(7):2399-2410.

70. Pedraza DF. Perfil antropométrico de criancas segundo a estrutura das creches. Cien Saude Colet 2017; 22(4):1361-1371.

71. Oliveira FC, Cotta RM, Sant'Ana LF, Priore SE, Franceschini SC. Programa Bolsa Familia e estado nutricional infantil: desafios estratégicos. Cien Saude Colet 2011; 16(7):3307-3316.
72. Oliosa PR, Zaniqueli DDA, Barbosa MCR, Mill JG. Relação entre composição corporal e dislipidemias em crianças e adolescentes. Cien Saude Colet 2019; 24(10):3743-3752.

73. Barbosa Filho VC, Campos W, Fagundes RR, Lopes AS, Souza EA. Presença isolada e combinada de indicadores antropométricos elevados em crianças: prevalência e fatores sociodemográficos associados. Cien Saude Colet 2016; 21(1):213-224.

74. Jesus GM, Castelao ES, Vieira TO, Gomes DR, Vieira GO. Déficit nutricional em crianças de uma cidade de grande porte do interior da Bahia, Brasil. Cien Saude Colet 2014; 19(5):1581-1588.

75. Kneipp C, Habitzreuter F, Mezadri T, Hofelmann DA. Excesso de peso e variáveis associadas em escolares de Itajaí, Santa Catarina, Brasil. Cien Saude Colet 2015; 20(8):2411-2422.

76. Lang RM, Almeida CC, Taddei JA. Segurança alimentar e nutricional de crianças menores de dois anos de famílias de trabalhadores rurais Sem Terra. Cien Saude Colet 2011; 16(7):3111-3118.

77. Mantovani SAS, Ramalho AA, Pereira FLCC, Oliart-Guzmán H, Delfino BM, Braña AM, Martins AC, Filgueira-Júnior JA, Santos AP. Nanismo em crianças menores de cinco anos de idade ainda e um problema de saúde na Amazônia Ocidental Brasileira: um estudo de base populacional em Assis Brasil, Acre, Brasil. Cien Saude Colet 2016; 21(7):2257-2266.

78. Orellana JDY, Marrero L, Alves CLM, Ruiz CMV, Hacon SS, Oliveira MW, Basta PC. Associação de baixa estatura severa em crianças indígenas Yanomami com baixa estatura materna: indícios de transmissão intergeracional. Cien Saude Colet 2019; 24(5):1875-1883.

79. Pedraza DF, Queiroz DD, Paiva AA, Cunha MA, Lima ZN. Seguridad alimentaria, crecimiento y niveles de vitamina $\mathrm{A}$, hemoglobina y zinc en ninos preescolares del nordeste de Brasil. Cien Saude Colet 2014; 19(2):641-650.

80. Pedraza DF, Sales MC, Menezes TN. Fatores associados ao crescimento linear de crianças socialmente vulneráveis do Estado da Paraíba, Brasil. Cien Saude Colet 2016; 21(3):935-946.

81. Rissin A, Figueiroa JN, Benicio MH,Batista Filho M. Retardo estatural em menores de cinco anos: um estudo "baseline". Cien Saude Colet 2011; 16(10):40674076.

82. Santos DFBD, Strapasson GC, Golin SDP, Gomes EC, Wille GMFC, Barreira SMW. Implicações da pouca preocupação e percepção familiar no sobrepeso infantil no município de Curitiba, PR, Brasil. Cien Saude Colet 2017; 22(5):1717-1724.

83. Pedraza DF, Menezes TN. Fatores de risco do déficit de estatura em crianças pré-escolares: estudo casocontrole. Cien Saude Colet 2014; 19(5):1495-1502.

84. Meller FO, Araújo CL, Madruga SW. Fatores associados ao excesso de peso em crianças brasileiras menores de cinco anos. Cien Saude Colet 2014; 19(3):943955.

85. Chaves CRMM, Cunha ALP, Costa AC, Costa RSS, Lacerda SV. Estado nutricional e distribuição de gordura corporal em crianças e adolescentes com fibrose cística. Cien Saude Colet 2015; 20(11):3319-3328.

86. Ferrari GLM, Solé D, Pires C, Matsudo V, Katzmarzyk PT, Fisberg M. Correlatos da gordura corporal e circunferência da cintura em crianças de São Caetano do Sul, Brasil. Cien Saude Colet 2019; 24(11):4019-4030. 
87. Jensen NSO, Camargo TFB, Bergamaschi DP. Índice de massa corpórea e perímetro da cintura são bons indicadores para classificação do estado nutricional de crianças. Cien Saude Colet 2016; 21(4):1175-1180.

88. Kuhn-Santos RC, Suano-Souza FI, Puccini RF, Strufaldi MWL. Fatores associados ao excesso de peso e baixa estatura em escolares nascidos com baixo peso. Cien Saude Colet 2019; 24(2):361-370.

89. Fonseca PCA, Carvalho CA, Ribeiro SAV, Nobre LN, Pessoa MC, Ribeiro AQ, Priore SE, Franceschini SDCC. Determinantes da velocidade média de crescimento de crianças até seis meses de vida: um estudo de coorte. Cien Saude Colet 2017; 22(8):2713-2726.

90. Caminha MFC, Azevedo PT, Sampaio BB, Acioly VM, Belo MP, Lira PI, Batista Filho M. Aleitamento materno em crianças de 0 a 59 meses no estado de Pernambuco, Brasil, segundo o peso ao nascer. Cien Saude Colet 2014; 19(7):2021-2032.

91. Caminha MFC, Serva VB, Anjos MM, Brito RB, Lins MM, Batista Filho M. Aleitamento materno exclusivo entre profissionais de um Programa Saúde da Família. Cien Saude Colet 2011; 16(4):2245-2250.

92. Méio MDBB, Villela LD, Gomes Junior SS, Tovar CM, Moreira MEL. Amamentação em lactentes nascidos pré-termo após alta hospitalar: acompanhamento durante o primeiro ano de vida. Cien Saude Colet 2018; 23(7):2403-2412.

93. Santos EMD, Silva LSD, Rodrigues BFS, Amorim TMAX, Silva CSD, Borba JMC, Tavares FCLP. Avaliação do aleitamento materno em crianças até dois anos assistidas na atenção básica do Recife, Pernambuco, Brasil. Cien Saude Colet 2019; 24(3):1211-1222.

94. Boccolini CS, Boccolini PM, Carvalho ML, Oliveira MI. Padrões de aleitamento materno exclusivo e internação por diarreia entre 1999 e 2008 em capitais brasileiras. Cien Saude Colet 2012; 17(7):1857-1863.

95. Gusmao AM, Beria JU, Gigante LP, Leal AF, Schermann LB. Prevalência de aleitamento materno exclusivo e fatores associados: estudo transversal com mães adolescentes de 14 a 16 anos em Porto Alegre, RS, Brasil. Cien Saude Colet 2013; 18(11):3357-3368.

96. Silva MA, Soares MM, Fonseca PCA, Vieira SA, Carvalho CA, Amaral RM, Franceschini SDCC, Novaes JF. Relação entre os tipos de aleitamento materno e o consumo de vitamina A e ferro em crianças de 6 a 12 meses. Cien Saude Colet 2019; 24(11):4009-4018.

97. Alves JS, Oliveira MIC, Rito RVVF. Orientações sobre amamentação na atenção básica de saúde e associação com o aleitamento materno exclusivo. Cien Saude Colet 2018; 23(4):1077-1088.

98. Silva CME, Pellegrinelli ALR, Pereira SCL, Passos IR, Santos LCD. Práticas educativas segundo os "Dez passos para o sucesso do aleitamento materno" em um Banco de Leite Humano. Cien Saude Colet 2017; 22(5):1661-1671.

99. Almeida GG, Spiri WC, Juliani CM, Paiva BS. Proteção, promoção e apoio ao aleitamento materno em um hospital universitário. Cien Saude Colet 2008; 13(2):487-494

100. Morgado CMC, Werneck GL, Hasselmann MH. Rede e apoio social e práticas alimentares de crianças no quarto mês de vida. Cien Saude Colet 2013; 18(2):367376.
101. Carrascoza KC, Possobon RF, Ambrosano GM, Costa Junior AL, Moraes AB. Determinantes do abandono do aleitamento materno exclusivo em crianças assistidas por programa interdisciplinar de promoção a amamentação. Cien Saude Colet 2011; 16(10):41394146.

102. Machado AK, Elert VW, Pretto AD, Pastore CA. Intenção de amamentar e de introdução de alimentação complementar de puérperas de um Hospital-Escola do sul do Brasil. Cien Saude Colet 2014; 19(7):19831989.

103. Fernandes RC, Höfelmann DA. Intenção de amamentar entre gestantes: associação com trabalho, fumo e experiência prévia de amamentação. Cien Saude Colet 2020; 25(3):1061-1072.

104. Tenorio MCDS, Mello CS, Oliveira ACM. Fatores associados a ausência de aleitamento materno na alta hospitalar em uma maternidade pública de Maceió, Alagoas, Brasil. Cien Saude Colet 2018; 23(11):35473556.

105. Mendes SC, Lobo IKV, Sousa SQ, Vianna RPT. Fatores relacionados com uma menor duração total do aleitamento materno. Cien Saude Colet 2019; 24(5):18211829.

106. Ferreira HLOC, Oliveira MF, Bernardo EBR, Almeida PC, Aquino PS, Pinheiro AKB. Fatores associados a adesão ao aleitamento materno exclusivo. Cien Saude Colet 2018; 23(3):683-690.

107. Rigotti RR, Oliveira MIC, Boccolini CS. Associação entre o uso de mamadeira e de chupeta e a ausência de amamentação no segundo semestre de vida. Cien Saude Colet 2015; 20(4):1235-1244.

108. Albuquerque SS, Duarte RC, Cavalcanti AL, Beltrão EM. A influência do padrão de aleitamento no desenvolvimento de hábitos de sucção não nutritivos na primeira infância. Cien Saude Colet 2010; 15(2):371378.

109. Moimaz SA, Rocha NB, Garbin AJ, Saliba O. Relação entre aleitamento materno e hábitos de sucção não nutritivos. Cien Saude Colet 2011; 16(5):2477-2484.

110. Mota TT, Caminha MFC, Figueiroa JN, Lira PI, Batista Filho M. Influência do aleitamento materno na hospitalização de menores de dois anos no estado de Pernambuco, Brasil, em 1997 e 2006. Cien Saude Colet 2015; 20(8):2347-2358.

111. Carvalho CA, Fonseca PCA, Nobre LN, Silva MA, Pessoa MC, Ribeiro AQ, Priore SE, Franceschini SDCC Fatores sociodemográficos, perinatais e comportamentais associados aos tipos de leite consumidos por crianças menores de seis meses: coorte de nascimento. Cien Saude Colet 2017; 22(11):3699-3710.

112. Bortolini GA, Giugliani ERJ, Gubert MB, Santos LMP. Amamentação está associada à diversidade alimentar infantil no Brasil. Cien Saude Colet 2019; 24(11):43454354.

113. von Seehausen MP, Oliveira MIC, Boccolini CS. Fatores associados ao aleitamento cruzado. Cien Saude Colet 2017; 22(5):1673-1682.

114. Oliveira CSM, Augusto RA, Muniz PT, Silva SA, Cardoso MA. Anemia e deficiência de micronutrientes em lactentes atendidos em unidades básicas de saúde em Rio Branco, Acre, Brasil. Cien Saude Colet 2016; 21(2):517-530 
115. Paula WK, Caminha MF, Figueiroa JN, Batista Filho M. Anemia e deficiência de vitamina A em crianças menores de cinco anos assistidas pela Estratégia Saúde da Família no Estado de Pernambuco, Brasil. Cien Saude Colet 2014; 19(4):1209-1222.

116. Oliveira TSC, Silva MC, Santos JN, Rocha DS, Alves CR, Capanema FD, Lamounier JA. Anemia entre pré -escolares: um problema de saúde pública em Belo Horizonte, Brasil. Cien Saude Colet 2014; 19(1):59-66.

117. Pedraza DF, Rocha AC, Sousa CP. Crescimento e deficiências de micronutrientes: perfil das crianças assistidas no núcleo de creches do governo da Paraíba, Brasil. Cien Saude Colet 2013; 18(11):3379-3390.

118. Miglioli TC, Fonseca VM, Gomes Junior SC, Lira PI, Batista Filho M. Deficiência de Vitamina A em mães e filhos no estado de Pernambuco. Cien Saude Colet 2013; 18(5):1427-1440.

119. Mariath AB, Giachini RM, Lauda LG, Grillo LP. Estado de ferro e retinol sérico entre crianças e adolescentes atendidos por equipe da Estratégia de Saúde da Família de Itajaí, Santa Catarina. Cien Saude Colet 2010; 15(2):509-516.

120. Pedraza DF, Araujo EMN, Santos GLDD, Chaves LRM, Lima ZN. Factores asociados a las concentraciones de hemoglobina en preescolares. Cien Saude Colet 2018; 23(11):3637-3647.

121. Oliveira AP, Pascoal MN, Santos LC, Pereira SC, Justino LE, Petarli GB, Kitoko PM. Prevalência de anemia e sua associação com aspectos sociodemográficos e antropométricos em crianças de Vitória, Espirito Santo, Brasil. Cien Saude Colet 2013; 18(11):3273-3280.

122. Souza NA, Vieira SA, Fonseca PCA, Andreoli CS, Priore SE, Franceschini SDCC. Dislipidemia familiar e fatores associados a alterações no perfil lipídico em crianças. Cien Saude Colet 2019; 24(1):323-332.

123. Arimatea JE, Silva CMFP, Costa AJL, Fonseca SC, Gama SGN, Lacerda EMA, Kale PL. Baixo peso ao nascer e peso pós-natal em bebês a termo menores de seis meses de idade, Rio de Janeiro, RJ, Brasil. Cien Saude Colet 2015; 20(5):1459-1466.

124. Lima MC, Oliveira GS, Lyra CO, Roncalli AG, Ferreira MA. A desigualdade espacial do baixo peso ao nascer no Brasil. Cien Saude Colet 2013; 18(8):2443-2452.

125. Belfort GP, Santos MMAS, Pessoa LDS, Dias JR, Heidelmann SP, Saunders C. Determinantes do baixo peso ao nascer em filhos de adolescentes: uma análise hierarquizada. Cien Saude Colet 2018; 23(8):26092620.

126. Capelli JC, Pontes JS, Pereira SE, Silva AA, Carmo CN, Boccolini CS, Almeida MF. Peso ao nascer e fatores associados ao período pre-natal: um estudo transversal em hospital maternidade de referência. Cien Saude Colet 2014; 19(7):2063-2072.

127. Fernandes JC, Nozawa MR. Estudo da efetividade de um programa de triagem auditiva neonatal universal. Cien Saude Colet 2010; 15(2):353-361.

128. Lessa CCR, Lamy Filho F, Lamy ZC, Silva AAM, Moreira MEL, Gomes MASM. Prevalência e fatores associados ao uso de surfactante em unidades de Terapia Intensiva Neonatais brasileiras: análise multinível. Cien Saude Colet 2018; 23(9):3067-3076.

129. Araújo CL, Rios CT, Santos MH, Gonçalves AP. Método Mãe Canguru: uma investigação da prática domiciliar. Cien Saude Colet 2010; 15(1):301-307.
130. Cesar JA, Acevedo JD, Kaczan CR, Venzo JCP, Costa LR, Silva L, Neumann NA. Intenção das mães em colocar o bebê para dormir em decúbito dorsal: um estudo de base populacional. Cien Saude Colet 2018; 23(2):501-508.

131. Pinheiro JMF, Tinoco LS, Rocha ASS, Rodrigues MP, Lyra O, Ferreira MAF. Atenção à criança no período neonatal: avaliação do pacto de redução da mortalidade neonatal no Rio Grande do Norte, Brasil. Cien Saude Colet 2016; 21(1):243-252.

132. Balbi B, Carvalhaes MABL, Parada CMGL. Tendência temporal do nascimento pré-termo e de seus determinantes em uma década. Cien Saude Colet 2016; 21(1):233-241.

133. Martini JA, Perosa GB,Padovani FHP. Qualidade de vida de escolares nascidos prematuros, o relato do cuidador e o auto-relato infantil. Cien Saude Colet 2019; 24(12):4699-4706.

134. Santos PC, Silva ZP, Chiaravalloti Neto F, Almeida MF. Análise espacial dos aglomerados de nascimentos ocorridos em hospitais SUS e não SUS do municipio de São Paulo, Brasil. Cien Saude Colet 2014; 19(1):235-244.

135. Cardoso ARP, Araújo MAL, Cavalcante MS, Frota MA, Melo SP. Análise dos casos de sífilis gestacional e congênita nos anos de 2008 a 2010 em Fortaleza, Ceará, Brasil. Cien Saude Colet 2018; 23(2):563-574.

136. Hebmuller MG, Fiori HH, Lago EG. Gestações subsequentes em mulheres que tiveram sífilis na gestação. Cien Saude Colet 2015; 20(9):2867-2878.

137. Lima MG, Santos RF, Barbosa GJ, Ribeiro GS. Incidência e fatores de risco para sífilis congênita em Belo Horizonte, Minas Gerais, 2001-2008. Cien Saude Colet 2013; 18(2):499-506.

138. Teixeira LO, Belarmino V, Goncalves CV, MendozaSassi RA. Tendência temporal e distribuição espacial da sífilis congênita no estado do Rio Grande do Sul entre 2001 e 2012. Cien Saude Colet 2018; 23(8):25872597.

139. Leivas PHS, Tejada CAO, Bertoldi AD, Santos AMA, Jacinto PA. Associação da posição socioeconômica e da depressão materna com a saúde das crianças: avaliação da PNAD 2008, Brasil. Cien Saude Colet 2018; 23(5):1635-1645.

140. Costa DO, Souza FIS, Pedroso GC, Strufaldi MWL. Transtornos mentais na gravidez e condições do recém-nascido: estudo longitudinal com gestantes assistidas na atenção básica. Cien Saude Colet 2018; 23(3):691-700.

141. Ribeiro DG, Perosa GB, Padovani FH. Fatores de risco para o desenvolvimento de crianças atendidas em Unidades de Saúde da Família, ao final do primeiro ano de vida: aspectos sociodemográficos e de saúde mental materna. Cien Saude Colet 2014; 19(1):215226.

142. Silva MLD, Cavalcante LIC, Heumann S, Lima TVR. Relação entre gênero e desempenho neuropsicomotor de crianças em Belém, Brasil. Cien Saude Colet 2018; 23(8):2721-2730.

143. Cavalcante MCV, Lamy FF, Franca AKTDC, Lamy ZC. Relação mãe-filho e fatores associados: anáise hierarquizada de base populacional em uma capital do Brasil-Estudo BRISA. Cien Saude Colet 2017; 22(5):16831693. 
144. Lamy Filho F, Medeiros SM, Lamy ZC, Moreira ME. Ambiente domiciliar e alterações do desenvolvimento em crianças de comunidade da periferia de São Luís MA. Cien Saude Colet 2011; 16(10):4181-4187.

145. Correia LL, Rocha HAL, Campos JS, Silveira DMI, Machado MMT, Leite AJM, Cunha AJLA. Interação entre suplementação de vitamina $\mathrm{A}$ e desnutrição crônica no desenvolvimento infantil. Cien Saude Colet 2019; 24(8):3037-3046.

146. Lourenco EC, Guerra LM, Tuon RA, Vidal e Silva SM, Ambrosano GM, Corrente JE, Cortellazzi KL, Vazquez FL, Meneghim MC, Pereira AC. Variáveis de impacto na queda da mortalidade infantil no Estado de São Paulo, Brasil, no período de 1998 a 2008. Cien Saude Colet 2014; 19(7):2055-2062.

147. Silva ESAD, Paes NA. Programa Bolsa Família e a redução da mortalidade infantil nos municípios do semiárido brasileiro. Cien Saude Colet 2019; 24(2):623630.

148. Rodrigues M, Bonfim C, Portugal JL, Frias PG, Gurgel IG, Costa TR, Medeiros Z. Análise espacial da mortalidade infantil e adequação das informações vitais: uma proposta para definição de áreas prioritárias. Cien Saude Colet 2014; 19(7):2047-2054.

149. Buhler HF, Ignotti E, Neves SM, Hacon SS. Análise espacial de indicadores integrados determinantes da mortalidade por diarreia aguda em crianças menores de 1 ano em regiões geográficas. Cien Saude Colet 2014; 19(10):4131-4140.

150. Oliveira GS, Lima MCBM, Lyra CO, Oliveira AGRC, Ferreira MAF. Desigualdade espacial da mortalidade neonatal no Brasil: 2006 a 2010. Cien Saude Colet 2013; 18(8):2431-2441.

151. Geib LT, Freu CM, Brandao M, Nunes ML. Determinantes sociais e biológicos da mortalidade infantil em coorte de base populacional em Passo Fundo, Rio Grande do Sul. Cien Saude Colet 2010; 15(2):363-370.

152. Sleutjes FCM, Parada CMGL, Carvalhaes MABL, Temer MJ. Fatores de risco de óbito neonatal em região do interior paulista, Brasil. Cien Saude Colet 2018; 23(8):2713-2720.

153. Lima JC, Mingarelli AM, Segri NJ, Zavala AA, Takano OA. Estudo de base populacional sobre mortalidade infantil. Cien Saude Colet 2017; 22(3):931-939.

154. Garcia LP, Santana LR. Evolução das desigualdades socioeconomicas na mortalidade infantil no Brasil, 1993-2008. Cien Saude Colet 2011; 16(9):3717-3728.

155. Santos HG, Andrade SM, Silva AM, Mathias TA, Ferrari LL, Mesas AE. Mortes infantis evitáveis por intervenções do Sistema Único de Saúde: comparação de duas coortes de nascimentos. Cien Saude Colet 2014; 19(3):907-916

156. Picoli RP, Cazola LHO, Nascimento DDG. Mortalidade infantil e classificação de sua evitabilidade por cor ou raça em Mato Grosso do Sul. Cien Saude Colet 2019; 24(9):3315-3324.

157. Tejada CAO, Triaca LM, Liermann NH, Ewerling F, Costa JC. Crises econômicas, mortalidade de crianças e o papel protetor do gasto público em saúde. Cien Saude Colet 2019; 24(12):4395-4404.

158. Melo MC, Ferraz RO, Nascimento JL, Donalisio MR Incidêcia e mortalidade por AIDS em crianças e adolescentes: desafios na região sul do Brasil. Cien Saude Colet 2016; 21(12):3889-3898.
159. Oliveira CM, Guimaraes MJB, Bonfim CVD, Frias PG, Antonino VCS, Guimaraes ALS, Medeiros ZM. Adequação da investigação dos óbitos infantis no Recife, Pernambuco, Brasil. Cien Saude Colet 2018; 23(3):701-714

160. Ramalho MO, Frias PG, Vanderlei LC, Macedo VC, Lira PI. Avaliação da incompletude da declaração de óbitos de menores de um ano em Pernambuco, Brasil, 1999-2011. Cien Saude Colet 2015; 20(9):2891-2898.

161. Silva LP, Moreira CM, Amorim MH, Castro DS, Zandonade E. Avaliação da qualidade dos dados do Sistema de Informações sobre Nascidos Vivos e do Sistema de Informações sobre Mortalidade no período neonatal, Espírito Santo, Brasil, de 2007 a 2009. Cien Saude Colet 2014; 19(7):2011-2020.

162. Maria LFBS, Araújo TVB. Um olhar sobre a vigilância dos óbitos fetais do Jaboatão dos Guararapes, Pernambuco, Brasil, em 2014. Cien Saude Colet 2017; 22(10):3415-3428.

163. Trombini ES, Schermann LB. Prevalência e fatores associados a adesão de crianças na terapia antirretroviral em três centros urbanos do sul do Brasil. Cien Saude Colet 2010; 15(2):419-425.

164. Araújo TM, Dantas JM, Carvalho CE, Costa MA. Surto de diarreia por rotavirus no município de Bom Jesus (PI). Cien Saude Colet 2010; 15(Supl. 1):10391046.

165. Torres RM, Bittencourt SA, Oliveira RM, Siqueira AS, Sabroza PC, Toledo LM. Uso de indicadores de nível local para análise espacial da morbidade por diarreia e sua relação com as condições de vida. Cien Saude Colet 2013; 18(5):1441-1450.

166. Venâncio TS, Tuan TS, Nascimento LFC. Incidência de tuberculose em crianças no estado de São Paulo, Brasil, sob enfoque espacial. Cien Saude Colet 2015; 20(5):1541-1547.

167. Caldart RV, Marrero L, Basta PC, Orellana JD. Fatores associados a pneumonia em crianças Yanomami internadas por condições sensíveis a atenção primária na região norte do Brasil. Cien Saude Colet 2016; 21(5):1597-1606.

168. Pereira EL, Bezerra JC, Brant JL, Araujo WN, Santos LMP. Perfil da demanda e dos Benefícios de Prestação Continuada (BPC) concedidos a crianças com diagnóstico de microcefalia no Brasil. Cien Saude Colet 2017; 22(11):3557-3566

169. Fernández RR, Larentis DZ, Fontana T, Jaeger GP, Moreira PB, Garcias GL, Roth MGM. Anencefalia: um estudo epidemiológico de treze anos na cidade de Pelotas. Cien Saude Colet 2005; 10(1):185-190.

170. Roncalli AG, Lima KC. Impacto do Programa Saúde da Família sobre indicadores de saúde da criança em municípios de grande porte da região Nordeste do Brasil. Cien Saude Colet 2006; 11(3):713-724.

171. Oliveira BLCA, Moreira JPL, Luiz RR. A influência da Estratégia Saúde da Família no uso de serviços de saúde por crianças no Brasil: análise com escore de propensão dos dados da Pesquisa Nacional de Saúde. Cien Saude Colet 2019; 24(4):1495-1505.

172. Mesquita Filho M, Luz BS, Araujo CS. A atenção primária a saúde e seus atributos: a situação das crianças menores de dois anos segundo suas cuidadoras. Cien Saude Colet 2014; 19(7):2033-2046. 
173. Vieira GO, Bastos MC, Reis MRD, Moreira ISS, Martins CDC, Gomes DR, Santana GS, Vieira TO. Fatores associados ao uso da Caderneta de Saúde da Criança em uma cidade de grande porte do nordeste brasileiro, 2009. Cien Saude Colet 2017; 22(6):1943-1954.

174. Vieira DS, Santos NCCB, Costa DKG, Pereira MM, Vaz EMC, Reichert APS. Registro de ações para prevenção de morbidade infantil na caderneta de saúde da criança. Cien Saude Colet 2016; 21(7):2305-2313.

175. Lobo IKV, Konstantyner T, Areco KCN, Vianna RPT, Taddei JAAC. Internações por Condições Sensíveis a Atenção Primaria de Menores de um ano, de 2008 a 2014, no estado de São Paulo, Brasil. Cien Saude Colet 2019; 24(9):3213-3226.

176. Moura EC, Moreira MCN, Menezes LA, Ferreira IA,Gomes R. Condições crônicas complexas em crianças e adolescentes: internações no Brasil, 2013. Cien Saude Colet 2017; 22(8):2727-2734.

177. Souza ECO, Santos ES, Rosa AM, Botelho C. Fatores socioeconômicos e risco para a hospitalização por asma em crianças em municípios de Mato Grosso, Brasil. Cien Saude Colet 2018; 23(8):2523-2532.

178. Vasconcelos MJOB, Rissin A, Figueiroa JN, Lira PIC, Batista Filho M. Doenças diarreicas e hospitalizações em menores de cinco anos no estado de Pernambuco, Brasil, nos anos de 1997 e 2006. Cien Saude Colet 2018; 23(3):715-722.

179. Moraes CG, Mengue SS, Tavares NU, Dal Pizzol TS. Utilização de medicamentos entre crianças de zero a seis anos: um estudo de base populacional no sul do Brasil. Cien Saude Colet 2013; 18(12):3585-3593.

180. Gentil LB, Robles AC, Grosseman S. Uso de terapias complementares por mães em seus filhos: estudo em um hospital universitário. Cien Saude Colet 2010; 15(Supl. 1):1293-1299.

181. Cavalcante LI, Magalhaes CM, Pontes FA. Processos de saúde e doença entre crianças institucionalizadas: uma visão ecológica. Cien Saude Colet 2009; 14(2):615-625.

182. Oliveira CB, Frechiani JM, Silva FM, Maciel EL. As ações de educação em saúde para crianças e adolescentes nas unidades básicas da região de Maruípe no município de Vitória. Cien Saude Colet 2009; 14(2):635-644.

183. Oliveira TB, Presoto LH. Eficácia de um programa de promoção da saúde em infantes de pré-escola na cidade de Anapolis, Goias. Cien Saude Colet 2009; 14(5):1891-1902.

184. Nunes ED. A revista Cien Saude Colet e o processo de institucionalização de um campo de conhecimentos e práticas. Cien Saude Colet 2015; 20(7):1975-1982.

185. Brasil. Ministério da Saúde (MS). Portaria no 1.067, de 4 de julho de 2005. Institui a Política Nacional de Atenção Obstétrica e Neonatal, e dá outra providências. Diário Oficial da União 2005; 5 jul.
186. Brasil. Ministério da Saúde (MS). Portaria no 1.459 , de 24 de junho de 2011. Institui, no âmbito do Sistema Único de Saúde - SUS - a Rede Cegonha. Diário Oficial da União 2011; 25 jun.

187. Brasil. Ministério da Saúde (MS). Portaria no 1.130 , de 5 de agosto de 2015. Institui a Política Nacional de Atenção Integral à Saúde da Criança (PNAISC) no âmbito do Sistema Único de Saúde (SUS). Diário Oficial da União 2015; 6 ago.

188. Brasil. Ministério da Saúde (MS). Política Nacional de Alimentação e Nutrição. Brasília: MS; 2012.

189. Brasil. Ministério da Saúde (MS). Guia alimentar para a população Brasileira. Brasília: MS; 2014.

190. Brasil. Ministério da Saúde (MS). Política Nacional de Atenção Básica. Brasília: MS; 2012.

191. Brasil. Ministério da Saúde (MS). Saúde da criança: aleitamento materno e alimentação complementar. Brasília: MS; 2015.

192. Brasil. Ministério da Saúde (MS). Núcleo de Apoio à Saúde da Família. Brasília: MS; 2014.

193. Leta J. Brazilian growth in the mainstream science: The role of human resources and national journals. $J$ Scientometric Res 2012; 1(1):44-52.

194. Barbosa MMAL, Cuenca AMB, Oliveira K, França Junior I, Alvarez MCA, Omae LY. Most-cited public health articles of scientific journals from Brazil. Rev Saude Publica 2019; 53:81.

195. Barros AJD, Victora CG,Wehrmeister FC. Desigualdades em saúde materno-infantil no Brasil. Pelotas: UFPel; 2019.

196. Jorge MHPM, Laurenti R, Gotlieb SLD. Análise da qualidade das estatísticas vitais brasileiras: a experiência de implantação do SIM e do SINASC. Cien Saude Colet 2007; 12(3):643-654.

197. Organização Pan-Americana da Saúde (OPAS). Indicadores de Saúde: Elementos conceituais e práticos. Santiago: OPAS; 2018.

Artigo apresentado em 08/06/2020

Aprovado em 24/06/2020

Versão final apresentada em 26/06/2020 